\title{
DETERMINANTES DA DIVERSIDADE DE GÊNERO DAS EMPRESAS DO BRICS
}

\section{DETERMINANTS OF THE GENDER DIVERSITY OF BRICS COMPANIES}

\author{
FRANCISCA EVERLENE NOGUEIRA PESSOA \\ Universidade Federal do Ceará (UFC) \\ Mestre em Administração e Controladoria (UFC) \\ Orcid: https://orcid.org/0000-0002-0029-0349 / E-mail:_everlenepessoa@gmail.com \\ ANA JENIFFER REBOUÇAS MAIA \\ Universidade Federal do Ceará (UFC) \\ Doutoranda em Administração e Controladoria (UFC) \\ Orcid: https://orcid.org/0000-0003-2125-5953 / E-mail: anajeniffer@gmail.com \\ Av. da Universidade, 2431 - Benfica, Fortaleza - CE, CEP: 60020-180.

\section{MAGALI CARVALHO FAÇANHA} \\ Universidade Federal do Ceará (UFC) \\ Mestre em Administração e Controladoria (UFC) \\ Orcid: https://orcid.org/0000-0002-6000-501X / E-mail: magali.facanha@hotmail.com

\section{DANIEL BARBOZA GUIMARÃES} \\ Professor na Universidade Federal do Ceará (UFC) \\ Doutor em Economia (UFC) \\ Orcid: https://orcid.org/0000-0001-6966-7194 / E-mail: barbozadan@hotmail.com

\section{SILVIA MARIA DIAS PEDRO REBOUÇAS} \\ Professora na Universidade Federal do Ceará (UFC) \\ Pós-Doutora em Métodos Quantitativos Aplicados à Economia e à Gestão (UFC) \\ Orcid: https://orcid.org/0000-0002-8475-9748 / E-mail: smdpedro@gmail.com
}

Submissão: 28/06/2019. Revisão: 07/07/2020. Aceite: 23/07/2020. Publicação: 03/08/2020.

DOI: http://dx.doi.org/10.22277/rgo.v13i3.4961

\section{RESUMO}

Considerando o crescente envolvimento das mulheres nos negócios e consequente representação feminina nas empresas, o estudo proporciona uma análise cross-national da participação feminina nas organizações, investigando a diversidade de gênero nas empresas tanto pela ótica da governança corporativa quanto pela da gestão. Com metodologia quantitativa, descritiva e documental, foram realizados testes de estatística descritiva, análise de variância, correlação de Pearson e regressão linear múltipla em uma amostra composta por 611 empresas do BRICS. Para a diversidade de gênero, variável dependente, foi considerada a participação feminina na governança corporativa, representada pelo conselho de administração, e a gestão das empresas, representada pela alta administração. As variáveis independentes consistiram nos fatores relacionados ao conselho de administração, tamanho da empresa, rentabilidade e cultura nacional, nas suas dimensões individualismo e masculinidade. Verificou-se baixa representatividade feminina nas empresas, sinalizando que as mulheres têm menor participação do que os homens na estrutura de poder das empresas e constatou-se diferenças significativas na participação feminina das empresas entre os países. Ainda, os resultados apontam a rentabilidade e a cultura nacional, representada pelo individualismo e a masculinidade, como fatores determinantes da diversidade de gênero das empresas da amostra. Assim, o trabalho põe em evidência a diversidade de gênero e contribui para a discussão acerca dos fatores determinantes da participação feminina no conselho de administração e na alta administração das empresas, fornecendo evidências empíricas da análise para países de economias emergentes.

Palavras-chave: Diversidade de gênero. Conselho de administração. Alta administração. 


\section{ABSTRACT}

Considering the growing involvement of women in business and the consequent female representation in companies, the study provides a cross-national analysis of women's participation in organizations, investigating gender diversity in companies both from the perspective of corporate governance as well as from the management perspective. With quantitative, descriptive, and documentary methodology, tests of descriptive statistics, analysis of variance, Pearson correlation and multiple linear regression were performed in a sample composed of 611 BRICS companies. For gender diversity, a dependent variable, the participation of women in corporate governance, represented by the board of directors, and corporate management, represented by senior management, was considered. The independent variables consisted of factors related to the board of directors, company size, profitability, and national culture, in their individualism and masculinity dimensions. There was a low female representation in companies, indicating that women have less participation than men in the corporate power structure and there were significant differences in the female participation of companies between countries. Furthermore, the results point to profitability and national culture, represented by individualism and masculinity, as determinants of the gender diversity of the sample companies. Thus, the work highlights gender diversity and contributes to the discussion about the determinants of female participation in the board of directors and in top management, providing empirical evidence of the analysis for countries in emerging economies.

Keywords: Gender diversity. Administrative Council. High administration.

\section{INTRODUÇÃO}

A diversidade pode ser conceituada como qualquer diferença relevante que particulariza um indivíduo de outro (MEISSNER; VERTOVEC, 2015). Pesquisas sobre diversidade a distinguem, geralmente, de duas formas: a observável e a não observável, sendo a diversidade observável aquela que se refere ao gênero, a idade, a raça e a etnia, enquanto a não observável é exemplificada pelo conhecimento, educação, valores, percepção, carinho e características de personalidade (BERNILE; BHAGWAT; YONKER, 2018; PELLED, 1996; SOLAKOGLU; DEMIR, 2016).

Os estudos sobre a diversidade relatam que manter diferentes opiniões torna as equipes diversas e as fazem tomar decisões de melhor qualidade, com pontos de vista de grupos sub-representados sendo expostos, estimulando a consideração de soluções não óbvias (HOFHUIS; ZEE; OTTEN, 2015). Em geral, a heterogeneidade na tomada de decisões e nos estilos de resolução de problemas gera melhores decisões por meio da operação de uma ampla gama de perspectivas e uma análise mais aprofundada e crítica dos problemas (PERRYMAN; FERNANDO; TRIPATHY, 2016). No Brasil, apenas recentemente a diversidade tem recebido maior atenção, em função, principalmente, do cenário de competitividade, inserção de empresas multinacionais, fusões e aquisições (GONÇALVES et al., 2015).

Nesse contexto, uma mudança significativa nas organizações tem sido a diversificação da força de trabalho (JOGULU; WOOD, 2008). Nas últimas décadas, aconteceram expressivas mudanças no padrão de introdução da mulher no mercado de trabalho e, acompanhando esta tendência, as mulheres estão, progressivamente, aumentando sua presença no ambiente corporativo (FORTIN, 2015). 
Segundo Carrasco et al. (2015), a diversidade de gênero tem se transformado em uma questão ética relevante para as organizações e vem sendo estimulada nas leis e códigos de governança nacional de diversos países. Dessa forma, uma associação de alterações nas atitudes sociais e uma legislação de isonomia mais sólida parece ter tido algum reflexo na melhoria da participação das mulheres nos conselhos das organizações, conforme exposto em estudos no contexto dos EUA (ADAMS; FERREIRA, 2009; KANG; CHENG; GRAY, 2007).

Considerando as inúmeras vantagens relacionadas com a presença de mulheres nas organizações, tais como melhoria da tomada de decisão, influência no desempenho e no sucesso de uma organização (ARFKEN; BELLAR; HELMS, 2004; MAHADEO; SOOBAROYEN; HANUMAN, 2012), Lehn, Patro e Zhao (2009) afirmam ser necessário investigar os fatores que podem levar as entidades a nomearem um número mais expressivo de mulheres para os seus conselhos.

Dentre esses fatores, conjectura-se que podem estar relacionadas à diversidade de gênero nas organizações características institucionais, relativas ao tamanho e desempenho da empresa e de seus instrumentos de governança, e fatores culturais, tendo em vista que a cultura reflete os valores, atitudes e comportamentos dos participantes de uma sociedade (HOFSTEDE, 1980).

Dentre os fatores culturais, no contexto da diversidade de gênero, destacam-se duas dimensões propostas por Hofstede (1980), quais sejam: individualidade, que representa o grau de interdependência que uma sociedade mantém entre seus membros, e masculinidade, que está associada aos papéis tradicionais de gênero para homens e mulheres.

Assim, com base na contextualização exposta, o presente trabalho está conduzido a responder o seguinte questionamento: Quais os determinantes da diversidade de gênero das empresas do BRICS? A partir da problemática levantada, o objetivo geral da pesquisa reside em identificar os determinantes da diversidade de gênero em empresas do BRICS.

Para tanto, foi realizada pesquisa descritiva, documental e quantitativa, tendo como população as empresas pertencentes aos países integrantes do BRICS (Brasil, Rússia, Índia, China e África do Sul), totalizando uma amostra de 611 companhias. Foram aplicados, assim, testes de estatística descritiva, diferença de médias, correlação e regressão linear múltipla.

O presente trabalho justifica-se no contexto empresarial, levando em consideração o crescente envolvimento de mulheres nos negócios e na sociedade e consequente representação feminina nas empresas (BUCKALEW et al., 2012; BURKE, 1997), bem como no contexto acadêmico, contribuindo para a linha de pesquisa que se propõe a analisar quais fatores estão relacionados à participação feminina nas organizações.

Destaca-se como diferencial da pesquisa a análise da diversidade de gênero nas empresas, considerando não apenas a governança corporativa, representada pelo conselho de administração, mas também a participação feminina na alta administração da empresa.

Ademais, a análise nos países do BRICS possibilita a investigação dessa relação em países de economias emergentes e com características significativamente diferentes. Assim, a pesquisa realiza uma análise cross-national da participação feminina nas organizações.

\section{REVISÃO DE LITERATURA}

\subsection{DIVERSIDADE DE GÊNERO}

Homens e mulheres agem em consonância com estereótipos e crenças, originadas na divisão do trabalho, relacionadas ao papel social que ocupam (EAGLY, 2009). Tais crenças são 
relevantes determinantes de como os gerentes dirigem suas empresas (ARTHUR; HERDMAN; YANG, 2016). A teoria do papel social trata das diferenças e semelhanças entre homens e mulheres no comportamento social, tendo como princípio o fato destas surgirem principalmente da sua distribuição em papéis sociais (EAGLY; WOOD, 2016). Destarte, a diferenciação no mercado de trabalho entre homens e mulheres está firmada no pressuposto de que existe divergência na forma de trabalhar/liderar dos colaboradores de gêneros distintos (GULL et al., 2018; PATRÍCIO, 2014).

Contudo, as mulheres participam menos do que os homens na estrutura de poder das organizações, como o conselho de administração (CARRASCO et al., 2015). Mesmo que a quantidade de membros femininos em conselhos corporativos esteja aumentando ligeiramente, de acordo com Kakabdse, Nicolopoulou e Kakabadse (2015), poucas empresas recrutam mulheres de forma ativa. Assim, mulheres membros de conselhos estão aumentando numericamente, mas as mudanças são pequenas e incrementais (MATTIS, 2000).

Apesar de as mulheres serem pelo menos iguais aos homens em capacidade de liderança, ainda existe uma distribuição desigual dos empregos mais bem remunerados na administração (BUCKALEW et al., 2012). Isto pode ocorrer pela existência de um teto de vidro que impede o acesso das mulheres a cargos executivos (EZZEDEEN, BUDWORTH; BAKER, 2015).

O teto de vidro corresponde a barreiras invisíveis que impedem as mulheres de chegarem aos cargos da alta gerência tanto quanto os colegas do sexo masculino (BUCKALEW, 2012). Os estudiosos ofereceram uma variedade de teorias para explicar o fenômeno do teto de vidro (DREHER, 2003), dentre os quais o conflito entre trabalho e família, os estereótipos de gênero e a divisão sexual do trabalho (BUCKALEW et al., 2012; COOK; GLASS, 2013; EAGLY; WOOD, 2016).

O conflito entre trabalho e família leva à possibilidade das mulheres optarem por não se tornarem CEOs, devido ao sentimento de não conseguir lidar com o equilíbrio entre vida profissional e familiar com as rigorosas demandas que uma posição de CEO assume, e mesmo que as mulheres decidam ir para a alta gerência e estejam dispostas a lidar com o trabalho de um CEO e sua família simultaneamente, podem não receber a promoção devido às percepções externas do conflito entre família e trabalho (BUCKALEW et al., 2012). Ademais, a divisão do trabalho está subjacente aos papéis de gênero de cada sociedade, que são expectativas compartilhadas consensualmente sobre homens e mulheres (EAGLY; WOOD, 2016).

O fenômeno do teto de vidro merece mais atenção da pesquisa em face dos problemas que gera, tais como a inibição do desejo por parte das mulheres de alcançar a alta gerência, a falta de diversidade entre os membros da equipe que pode levar a decisões ruins e a redução da oferta de talentos e recursos necessários nas organizações como consequência das barreiras baseadas em gênero (DREHER, 2003).

Inúmeros benefícios da diversidade de gênero nas organizações foram evidenciados pela Comissão Europeia (2012), tais como: a melhoria do desempenho financeiro das empresas; as diferentes maneiras de encarar os mercados e de tomada de decisões por parte das mulheres; as melhorias éticas e do corporate governance; e o fato de as mulheres, em média, possuírem um maior nível de qualificação que os homens.

Nesse sentido, Lazzaretti e Godoi (2012) relataram que as características comportamentais tipicamente femininas tendem a exercer influência positiva sobre as dinâmicas dos conselhos de administração, oferecendo uma oportunidade para as empresas testarem a diversidade de ideias, além de desenvolverem suas políticas públicas (ARFKEN; BELLAR; HELMS, 2004). 
Nesse contexto, o aumento da presença feminina no mercado de trabalho muito se deve à pressão realizada por parte dos governos de diversos países para que as organizações convoquem um número maior de mulheres para os conselhos corporativos, particularmente para o conselho de administração (FARRELL; HERSCH, 2005; GREEN; HOMROY, 2018). Por exemplo, em 2003, a Noruega aprovou uma lei que exige que quarenta por cento de todos os membros da diretoria das empresas listadas fossem mulheres até 2008 (CATALYST, 2008). Segundo Carrasco et al. (2015), a adoção de tais leis ou diretrizes pode diminuir consideravelmente as desigualdades da participação feminina nas entidades entre países, porém, tais diferenças ainda são significativas.

Cabe avaliar, ainda, como as mulheres que obtiveram seus cargos de diretora no mercado competitivo de diretores ultrapassaram o teto de vidro, considerando que para tanto, as mulheres podem assumir os estereótipos de gêneros masculinos, adaptando seu comportamento para que as diferenças de gênero desapareçam (ADAMS; FUNK, 2012). Daí a necessidade da integração das políticas de gênero com vistas à eliminação da desigualdade entre os sexos, das disparidades salariais e da divisão sexual do trabalho (GÁMEZ; ANGELES, 2019).

No contexto da diversidade de gênero nas organizações, podem ser mencionados alguns estudos que trataram sobre a temática (ADAMS; FERREIRA, 2009; ADAMS; FUNK, 2012; CARRASCO et al., 2015; HUMPHRIES; WHELAN, 2017; TERJESEN; SEALY; SINGH, 2009).

Uma revisão de literatura foi feita por Terjesen, Sealy e Singh (2009) para examinar como a diversidade de gênero em conselhos corporativos influencia a governança corporativa, que, por sua vez, impacta a performance da firma. Os autores descreveram a pesquisa sobre perspectivas teóricas, analisando características, determinantes e o impacto das mulheres nos conselhos corporativos. Os resultados indicaram que a participação feminina em conselhos trata de melhorar a governança corporativa através de um melhor uso do capital intelectual, bem como sobre a construção de instituições empresariais mais inclusivas e justas.

Em um estudo sobre a mesma temática, Carrasco et al. (2015) analisaram especificamente a nomeação de mulheres para conselhos de empresas para investigar a existência de um viés cultural. Os autores reuniram dados de 7.302 conselhos de 32 países para testar se houve implicações culturais usando as quatro dimensões principais de Hofstede (1980). Seus resultados indicaram que duas das dimensões, o poder de distância (PDI) e a masculinidade (MAS), afetaram negativamente a porcentagem de mulheres em conselhos.

Por sua vez, a pesquisa de Humphries e Whelan (2017) investigou a relação entre a cultura nacional e as melhores práticas como recomendado nos códigos de governança corporativa a nível nacional. Os autores utilizaram para mensurar a governança corporativa: independência do conselho, composição de gênero, liderança do conselho e frequência da reunião, e, para cultura nacional, as dimensões culturais de Hofstede (1980). Foram coletados códigos de governança corporativa de 55 países e os resultados apontaram relações significativas entre as dimensões culturais de Hofstede (1980) e as quatro características de governança corporativa examinadas no estudo.

Ainda, para uma amostra de empresas dos EUA, Adams e Ferreira (2009) analisaram o impacto da presença feminina nos insumos e nos resultados das empresas. Para o período de 1996 a 2003, os autores encontraram que as diretoras do sexo feminino têm registros de um melhor atendimento do que os diretores masculinos, que os diretores masculinos têm menos problemas de atendimento e que as mulheres são mais propensas a se juntar aos comitês de monitoramento. Ademais, o desempenho e os diretores recebem mais remuneração baseada em ações em empresas que possuem painéis mais diversos em termos de gênero. 
O estudo de Adams e Funk (2012) explorou as diferenças existentes entre homens e mulheres na administração das organizações no universo de diretores e CEOs (1.796 indivíduos) de empresas de capital aberto na Suécia em 2005. Os resultados indicaram que diretores homens e mulheres diferem sistematicamente em seus valores centrais e atitudes de risco, mas de maneiras que diferem das diferenças de gênero na população em geral, encontrando que as mulheres diretoras são mais benevolentes e preocupadas universalmente, mas menos orientadas pela tradição e segurança e menos avessas a riscos.

\subsection{FATORES RELACIONADOS COM A DIVERSIDADE DE GÊNERO}

Tendo em vista que a expansão da presença de mulheres no mercado de trabalho colaborou para a existência de uma maior diversidade de gênero no conselho de administração das organizações (PATRÍCIO, 2014), diversos estudos nacionais e internacionais propuseram-se a analisar os fatores que exercem influência na composição de conselhos de administração corporativos (ALI, 2018) e na presença de mulheres em cargos de liderança (TERJESEN; SEALY; SINGH, 2009).

Nesse sentido, ao servirem como instrumento de controle interno nas organizações, Elyasiani e Zhang (2015) destacam o papel dos conselhos de administração, sendo relevante o estudo da composição do mesmo e a realização de uma análise das distintas características que poderão exercer influência sobre o comportamento de tal órgão.

Pode-se constatar o aumento da participação feminina nos cargos importantes das organizações de forma generalizada (SILVA JUNIOR; MARTINS, 2017). As corporações estão começando a experimentar mudanças significativas nos conjuntos de candidatos potenciais para cargos de alto escalão (BURKE, 1997; BURKE; NELSON, 2002;). Assim, convém investigar as diferenças de gênero e a sua influência na atuação em cargos de liderança (NOGUEIRA; KUBO, 2013).

Dessa forma, ao analisar os fatores que exercem influência sobre a composição do conselho de administração, mais especificamente aqueles que podem afetar a presença feminina, destaca-se a variável tamanho do conselho como determinante da presença de mulheres no mesmo, de acordo com Erhardt, Werbel e Shrader (2003).

Em seu estudo, Hyland e Marcellino (2002) verificaram que existia uma relação positiva entre o tamanho do conselho de administração e o número de mulheres no órgão, sendo que as empresas com maior número de membros no conselho de administração possuíam, em média, uma maior participação feminina (LUOMA; GOODSTEIN, 1999). Assim, quanto maior o conselho de administração, maior o número de mulheres diretoras (BRAMMER; MILLINGTON; PAVELIN, 2007; HYLAND; MARCELLINO, 2002). Entretanto, alguns países limitam o número de diretores em seus conselhos (TERJESEN; SEALY; SINGH, 2009).

Diante do exposto, decorreu-se a primeira hipótese:

H1: O tamanho do conselho de administração se relaciona positivamente com a participação feminina no conselho de administração e na alta administração das empresas.

Um dos determinantes mais utilizados em pesquisas relacionadas com a diversidade de gênero é a dimensão da empresa (ADAMS; FERREIRA, 2007; CARTER; SIMKINS; SIMPSON, 2003). Waddock e Graves (1997) sugeriram que, à proporção que as empresas aumentam em tamanho, atraem mais atenção de comunidades externas e precisam responder mais abertamente às demandas das partes interessadas.

Nesse mesmo sentido, diversos autores (PFEFFER; SALANCIK, 1978; POWELL, 1991; WADDOCK; GRAVES, 1997) relataram que, em decorrência de seu tamanho, grandes organizações são mais visíveis e, portanto, estão propensas a uma maior atenção de grupos 
Francisca Everlene Nogueira Pessoa, Ana Jeniffer Rebouças Maia, Magali Carvalho Façanha, Daniel Barboza Guimarães e Silvia Maria Dias Pedro Rebouças

externos, como o estado, os meios de comunicação social, os grupos profissionais e o público em geral.

Dessa forma, as empresas de grande porte estão mais sujeitas às avaliações feitas pelo exterior e às exigências feitas pela sociedade, sendo natural que tais organizações procurem incluir um maior número de mulheres no conselho de administração (LI; CHEN, 2018; SALANCICK, 1979) e em conselhos corporativos (LUOMA; GOODSTEIN, 1999).

Isto posto, desenvolveu-se a segunda hipótese:

H2: O tamanho da empresa se relaciona positivamente com a participação feminina no conselho de administração e na alta administração das empresas.

Muitos autores buscam examinar a existência de relacionamento entre o desempenho ou rentabilidade e a participação de mulheres no conselho de administração. Erhardt, Werbel e Shrader (2003), Mahadeo, Soobaroyen e Hanuman (2012) e Lückerath-Rovers (2010), nos resultados de seus estudos, constataram uma relação positiva entre o desempenho e a participação de mulheres no conselho de administração.

Todavia, Adams e Ferreira (2009), ao analisar tal relação, encontraram uma relação negativa. Essas divergentes conclusões, no que diz respeito à relação entre o desempenho e a diversidade de gênero, podem estar associadas com variados fatores, tais como diferenças temporais, tamanho das empresas, metodologias utilizadas na pesquisa e o fato de se tratar de períodos econômicos diferentes analisados (GÓMEZ; LAFUENTE; VAILLANT, 2018).

Pode-se argumentar que as mulheres possuem uma tendência a atuar em conselhos de empresas de desempenho superior (FARRELL; HERSCH, 2005). Os autores sugerem que uma escassez de oferta possibilita que as mulheres auto selecionem as empresas, ou que essas organizações possam se concentrar mais em metas para diversificar seu quadro de colaboradores.

Considerando o exposto, elaborou-se a terceira hipótese:

H3: A rentabilidade se relaciona positivamente com a participação feminina no conselho de administração e na alta administração das empresas.

Ao discutir sobre os determinantes da diversidade de gênero, a literatura anterior se atém principalmente a diversos elementos dominantes, tais como o nível de desenvolvimento econômico, a governança corporativa e fatores específicos (DU, 2016). Entretanto, é inegável que a diversidade de gênero do conselho de administração sujeita-se a fatores culturais (PERETZ; LEVI; FRIED, 2015). Dessa forma, uma dimensão, levando em consideração as dimensões culturais de Hofstede (1980), que pode afetar a participação feminina nos conselhos de administração, é o individualismo.

Tal dimensão, de acordo com o website de Hofstede (2017), aborda o grau de interdependência que uma sociedade mantém entre seus membros, refletindo se a autoimagem das pessoas é definida em termos de "eu" ou "nós". Nas culturas de individualismo elevado, um conselho de administração pareceria mais legítimo se representasse os interesses de uma ampla gama de indivíduos ou partes interessadas (HUMPHRIES; WHELAN, 2017). Deste modo, a diversidade de gênero seria vista como uma consideração relevante na composição do conselho (HUMPHRIES; WHELAN, 2017).

Um alto valor nesta dimensão poderia espelhar um comprometimento com os direitos humanos individuais, que poderia abranger a igualdade de gênero com base no mérito pessoal (CARRASCO et al., 2015). Assim, pode ser plausível que o individualismo se manifeste em um anseio em perseguir as preferências particulares, independentemente de estereótipos, convenções, etc, ou seja, em um país caracterizado por um grau relativamente alto de 
individualismo, é mais provável que as mulheres procurem posições como a de conselho diretor, ainda de acordo com os autores.

Isto posto, desenvolveu-se a quarta hipótese:

H4: Uma cultura nacional de elevado individualismo se relaciona positivamente com a participação feminina no conselho de administração e na alta administração das empresas.

Ainda levando em consideração que pode haver um viés cultural na designação de mulheres para os conselhos de administração das empresas (CARRASCO et al., 2015), uma outra dimensão definida por Hofstede (1980) que pode influenciar a composição de gênero dos conselhos é a masculinidade. Tal dimensão, de acordo com o website de Hofstede (2017), representa uma preferência na sociedade pela realização, heroísmo, assertividade e recompensas materiais pelo sucesso.

Nas culturas de elevada masculinidade, os papéis de gênero são visivelmente determinados pelas normas sociais e em virtude de tal fato, é pouco provável que a composição de gênero nos conselhos de administração seja uma consideração em culturas masculinas (HUMPHRIES; WHELAN, 2017).

As sociedades tipicamente masculinas determinam os papéis de gênero de maneira tradicional e estereotipada (CARRASCO et al., 2015). Sealy, Doldor e Vinnicombe (2009) e Oakley (2000) fornecem evidências empíricas que a atribuição de valores estereotipados às mulheres pode influenciar de forma negativa a sua participação nos conselhos corporativos.

Assim, formulou-se a quinta hipótese da pesquisa:

H5: Uma cultura nacional de elevada masculinidade se relaciona negativamente com a participação feminina no conselho de administração e na alta administração das empresas.

\section{METODOLOGIA}

A metodologia da pesquisa classifica-se como descritiva, à medida em que buscou especificar as propriedades e características dos fenômenos estudados (SAMPIERI; COLLADO; LUCIO, 2013); documental, diante da utilização de documentos como fonte de dados (MARTINS; THEÓPHILO, 2009); e quantitativa, ao passo que os dados coletados foram quantificados e preparados para a aplicação de técnicas estatísticas (MARTINS; THEÓPHILO, 2009).

A população consiste nas empresas pertencentes aos países integrantes do BRICS (Brasil, Rússia, Índia, China e África do Sul). A amostra foi composta por 611 dentre as empresas do BRICS não financeiras com dados disponíveis na Bloomberg Database ${ }^{\circledR}$ em $21 / 11 / 2017$. Os dados foram coletados na Bloomberg Database ${ }^{\circledR}$ e no website de Hofstede (2017), tendo como base o exercício findo em 31/12/2016.

Para a participação feminina no conselho de administração e na alta administração, foram observadas a proporção de mulheres no conselho de administração e como executivas, respectivamente. As características capazes de afetar a diversidade de gênero nas empresas estão relacionadas ao conselho de administração, tamanho da empresa, rentabilidade e cultura nacional (individualismo e masculinidade). A Figura 1 evidencia as variáveis dependentes, independentes e de controle utilizadas no presente estudo, bem como suas respectivas proxies e referências. 
Francisca Everlene Nogueira Pessoa, Ana Jeniffer Rebouças Maia, Magali Carvalho Façanha, Daniel Barboza Guimarães e Silvia Maria Dias Pedro Rebouças

Quadro 1 - Variáveis dependentes, independentes e de controle

\begin{tabular}{|c|c|c|c|c|}
\hline & Variável & Base Teórica & Descrição & $\begin{array}{c}\text { Fonte de } \\
\text { Coleta }\end{array}$ \\
\hline \multirow{2}{*}{ Dependentes } & $\begin{array}{l}\text { Participação } \\
\text { feminina no } \\
\text { conselho de } \\
\text { administração }\end{array}$ & $\begin{array}{c}\text { Carrasco et al. (2015); } \\
\text { Humphries e Whelan, (2017); } \\
\text { Silva e Martins (2017) }\end{array}$ & $\begin{array}{c}\text { Proporção (\%) de mulheres } \\
\text { no conselho de } \\
\text { administração }\end{array}$ & $\begin{array}{l}\text { Bloomberg } \\
\text { Database }^{\circledR}\end{array}$ \\
\hline & $\begin{array}{l}\text { Participação } \\
\text { feminina na } \\
\text { alta } \\
\text { administração }\end{array}$ & $\begin{array}{c}\text { Adams e Ferreira (2009); Silva } \\
\text { e Margem (2015); Silveira et } \\
\text { al. (2014) }\end{array}$ & $\begin{array}{c}\text { Proporção (\%) de mulheres } \\
\text { executivas }\end{array}$ & $\begin{array}{l}\text { Bloomberg } \\
\text { Database }^{\circledR}\end{array}$ \\
\hline \multirow{5}{*}{ Independentes } & $\begin{array}{l}\text { Tamanho do } \\
\text { conselho }\end{array}$ & $\begin{array}{l}\text { Brammer, Millington e Pavelin } \\
\text { (2007); }\end{array}$ & $\begin{array}{c}\text { Número de integrante do } \\
\text { conselho de administração } \\
\text { das empresas }\end{array}$ & $\begin{array}{l}\text { Bloomberg } \\
\text { Database }^{\circledR}\end{array}$ \\
\hline & $\begin{array}{l}\text { Tamanho da } \\
\text { empresa }\end{array}$ & $\begin{array}{l}\text { Carter, Simkins e Simpson } \\
\text { (2003); Erhardt, Werbel e } \\
\text { Shrader (2003); Peng (2004) }\end{array}$ & $\begin{array}{l}\text { Logaritmo natural do total } \\
\text { de ativos }\end{array}$ & $\begin{array}{l}\text { Bloomberg } \\
\text { Database }^{\circledast}\end{array}$ \\
\hline & Rentabilidade & $\begin{array}{l}\text { Boulouta (2013); Du (2016); } \\
\text { Sila, Gonzalez e Hagendorf } \\
\text { (2016) }\end{array}$ & $\begin{array}{l}\text { Retorno sobre o } \\
\text { patrimônio líquido (ROE) }\end{array}$ & $\begin{array}{l}\text { Bloomberg } \\
\text { Database }^{\circledR}\end{array}$ \\
\hline & Masculinidade & $\begin{array}{c}\text { Carrasco et al. (2015); } \\
\text { Humphries e Whelan (2017) }\end{array}$ & $\begin{array}{c}\text { Preferência social pela } \\
\text { realização, assertividade e } \\
\text { recompensas materiais } \\
\text { pelo sucesso }\end{array}$ & $\begin{array}{l}\text { Website de } \\
\text { Hofstede }\end{array}$ \\
\hline & Individualismo & Humphries e Whelan (2017) & $\begin{array}{c}\text { Grau de interdependência } \\
\text { que uma sociedade } \\
\text { mantém entre seus } \\
\text { membros }\end{array}$ & $\begin{array}{l}\text { Website de } \\
\text { Hofstede }\end{array}$ \\
\hline \multirow{2}{*}{ Controle } & $\begin{array}{l}\text { Setor de } \\
\text { atuação }\end{array}$ & $\begin{array}{c}\text { Brammer, Millington e Pavelin } \\
\text { (2007); Erhardt, Werbel e } \\
\text { Shrader (2003); Nielsen e Huse } \\
\text { (2010) }\end{array}$ & $\begin{array}{c}\text { Setor da empresa de } \\
\text { acordo com o Padrão } \\
\text { Global de Classificação de } \\
\text { Indústria }\left(\text { GICS }^{\circledR}\right)\end{array}$ & $\begin{array}{l}\text { Bloomberg } \\
\text { Database }^{\circledR}\end{array}$ \\
\hline & $\begin{array}{l}\text { Sistema legal } \\
\text { do país }\end{array}$ & $\begin{array}{c}\text { La Porta, Lopes-de-Silanes e } \\
\text { Shleifer (1999); Santos e Viana } \\
\text { Jr. (2017) }\end{array}$ & $\begin{array}{c}\text { Dummy: } 1 \text {, se uma } \\
\text { empresa é de um país de } \\
\text { sistema legal common law } \\
\text { e } 0 \text {, caso contrário }\end{array}$ & $\begin{array}{l}\text { Juriglobe } \\
\text { (2017) }\end{array}$ \\
\hline
\end{tabular}

Fonte: Elaborada pelos autores.

Para uma visão geral do comportamento das variáveis investigadas, foram realizados testes de estatística descritiva. Na sequência, foram realizados testes de diferença de médias e correlação, a fim de se verificar semelhanças e diferenças entre as empresas, bem como verificar as primeiras correlações entre as variáveis.

Com foco no objetivo geral de investigar os determinantes da diversidade de gênero nas empresas dos cinco países analisados, foi aplicado o teste de Regressão Linear Múltipla, conforme evidencia a Equação 1:

WOMEN $_{i, j}=\alpha_{1}+\beta_{1}$ BSIZE $_{i, j}+\beta_{2}$ TAM $_{i, j}+\beta_{3}$ RENT $_{i, j}+\beta_{4} M_{\text {MAS }}{ }_{i, j}+\beta_{5} I D V_{i, j}+\beta_{6} S_{E T} T_{i, j}+\beta_{7} S_{S I S T}+\varepsilon_{i, j}$ (Equação 1)

Em que: WOMEN = participação feminina nas empresas; $B S I Z E$ = tamanho do conselho de administração; TAM = tamanho da empresa; RENT = rentabilidade; MAS = masculinidade; IDV = individualismo; SET = setor de atuação das empresas; SIST = Sistema legal; e $\varepsilon=$ termo de erro. 
A partir da Equação 1, foram testados dois modelos distintos. O Modelo 1 considerou a participação feminina no conselho de administração das empresas, enquanto o Modelo 2 utilizou a participação feminina na alta administração das empresas da amostra.

Para operacionalização dos testes mencionados, foram utilizados o software Statistical Package for the Social Sciences (SPSS), versão 22, estabelecendo-se o nível crítico de significância ( $p$-value) 0,05 como aceitável. Cabe destacar, ainda, a utilização do aplicativo Stata, versão 13, para verificação da homocedasticidade dos dados.

\section{ANÁLISE DOS RESULTADOS}

Quanto à participação feminina no conselho de administração e na alta administração das empresas, a Tabela 1 apresenta as estatísticas descritivas das empresas distribuídas por país.

Tabela 1 - Estatística descritiva da participação feminina por país

\begin{tabular}{|c|c|c|c|c|c|c|}
\hline $\begin{array}{c}\text { País/No de } \\
\text { observações }\end{array}$ & Variáveis & Mínimo & Máximo & Média & Desvio-padrão & CV \\
\hline África do Sul & Women_Board & 6,67 & 62,50 & 24,80 & 11,16 & 0,45 \\
\hline$N=47$ & Women_Executive & 0,00 & 66,67 & 16,07 & 15,74 & 0,98 \\
\hline Brasil & Women_Board & 0,00 & 33,33 & 6,66 & 8,69 & 1,30 \\
\hline $\mathrm{N}=97$ & Women_Executive & 0,00 & 66,67 & 8,12 & 11,72 & 1,44 \\
\hline China & Women_Board & 0,00 & 66,67 & 11,85 & 11,85 & 1,00 \\
\hline$N=359$ & Women_Executive & 0,00 & 100,00 & 13,22 & 15,00 & 1,13 \\
\hline Índia & Women_Board & 0,00 & 37,50 & 14,55 & 7,89 & 0,54 \\
\hline$N=84$ & Women_Executive & 0,00 & 50,00 & 6,70 & 10,38 & 1,55 \\
\hline Rússia & Women_Board & 0,00 & 26,67 & 4,70 & 7,67 & 1,63 \\
\hline$N=24$ & Women_Executive & 0,00 & 38,46 & 9,31 & 9,48 & 1,02 \\
\hline \multirow{2}{*}{ Total } & Women_Board & 0,00 & 66,67 & 12,11 & 11,60 & 0,96 \\
\hline & Women_Executive & 0,00 & 100,00 & 11,58 & 14,10 & 1,22 \\
\hline
\end{tabular}

Fonte: Elaborada pelos autores.

De acordo com os resultados da participação feminina nas empresas, evidenciados pela proporção de mulheres no conselho de administração e na alta administração, o país com maior participação feminina nas empresas é a África do Sul, com média de $24,80 \%$ de mulheres no conselho de administração e $16,07 \%$ de mulheres na alta administração das empresas, bem acima das médias de 12,11 e 11,58\%, respectivamente, da amostra.

Os países com menor diversidade de gênero nas empresas, por sua vez, foram a Rússia, quanto à participação feminina no conselho de administração, com média de $4,70 \%$ do conselho composto por mulheres, e a Índia, no que tange à participação feminina na alta administração, cuja média consistiu em $6,70 \%$.

Ademais, todos os países têm empresas sem participação feminina nas empresas, com exceção da África do Sul, cujo mínimo de participação feminina no conselho de administração é de 6,67\%. Tal resultado está alinhado ao encontrado por Liao, Luo e Tang (2015), que encontraram empresas sem participação feminina no conselho de administração no Reino Unido, bem como ao de Viana Jr. et al. (2017), que, de modo similar, encontraram empresas sem participação feminina ao pesquisar as empresas da Argentina, Brasil, Chile e México.

Observa-se a heterogeneidade dos dados, à medida em que apresentam alto desviopadrão (11,60 e 14,10 para a participação feminina no conselho e na alta administração das empresas da amostra, respectivamente). 
Ressalta-se, ainda, que as médias, em geral, da participação feminina nas empresas refletem uma baixa diversidade de gênero, corroborando Carrasco et al. (2015), que apontam que as mulheres têm menor participação do que os homens na estrutura de poder das empresas. Apesar dos autores argumentarem o crescimento da participação feminina nas empresas (DAVIDSON; BURKE, 2004; PATRÍCIO, 2014; SILVA JR.; MARTINS, 2017), os números de mulheres como membros do conselho estão aumentando, entretanto, as mudanças são pequenas e incrementais (MATTIS, 2000).

Ainda acerca das estatísticas descritivas, a Tabela 2 evidencia as características das variáveis independentes.

Tabela 2 - Estatística descritiva das variáveis independentes

\begin{tabular}{ccccccc}
\hline Variável & N & Mínimo & Máximo & Média & Desvio-padrão & CV \\
\hline BSIZE & 611 & 4,00 & 20,00 & 9,84 & 2,52 & 0,26 \\
TAM & 611 & 3,91 & 17,36 & 10,91 & 2,09 & 0,19 \\
RENT & 611 & $-124,08$ & 205,81 & 11,69 & 19,68 & 1,68 \\
MAS & 611 & 36,00 & 66,00 & 60,52 & 8,11 & 0,13 \\
IDV & 611 & 20,00 & 65,00 & 30,91 & 14,50 & 0,47 \\
\hline
\end{tabular}

Fonte: Elaborada pelos autores.

Em relação ao tamanho do conselho de administração, observa-se que este vai de 4 a 20 membros, com média de 10 membros, sendo os dados homogêneos, tendo em vista o desvio-padrão apresentado, de 2,52. Quanto ao tamanho da empresa, a variável apresenta homogeneidade, tendo em vista a linearização realizada por meio do cálculo do logaritmo natural do ativo total. No que tange à rentabilidade, por sua vez, mensurada pelo retorno sobre o patrimônio líquido, algumas empresas tiveram prejuízo, enquanto outras registraram elevada rentabilidade.

Para a cultura nacional, foram consideradas as dimensões masculinidade e individualismo, cujas pontuações são representadas numa escala de 0-100. Assim, os resultados evidenciam alta média de masculinidade $(60,52)$, que indica que há, nos países da amostra, preferência social pela realização, heroísmo, assertividade e recompensas materiais pelo sucesso. Já o individualismo apresenta média baixa $(30,91)$, sinalizando sociedades em que os indivíduos podem esperar que seus parentes ou membros de um grupo específico cuide deles em troca de lealdade inquestionável.

No intuito de verificar se as médias da participação feminina dos países investigados são estatisticamente diferentes, foi realizada uma análise de variância da proporção da participação feminina entre os países, como apresenta a Tabela 3.

Tabela 3 - Análise de variância (ANOVA): Participação feminina por país

\begin{tabular}{|c|c|c|c|c|c|}
\hline Variável & País & $\mathbf{N}$ & Média & $\mathbf{F}$ & Sig \\
\hline \multirow{5}{*}{ Conselho } & África do Sul & 47 & 24,80 & \multirow{5}{*}{26,68} & \multirow{5}{*}{0,00} \\
\hline & Brasil & 97 & 6,66 & & \\
\hline & China & 359 & 11,85 & & \\
\hline & Índia & 84 & 14,55 & & \\
\hline & Rússia & 24 & 4,70 & & \\
\hline \multirow{5}{*}{$\begin{array}{c}\text { Alta } \\
\text { administração }\end{array}$} & África do Sul & 47 & 16,07 & \multirow{5}{*}{6,79} & \multirow{5}{*}{0,00} \\
\hline & Brasil & 97 & 8,12 & & \\
\hline & China & 359 & 13,22 & & \\
\hline & Índia & 84 & 6,70 & & \\
\hline & Rússia & 24 & 9,31 & & \\
\hline
\end{tabular}

Fonte: Elaborada pelos autores. 
A partir dos resultados da Tabela 3, é possível inferir que as médias de participação feminina, tanto no conselho de administração quanto na alta administração das empresas, são estatisticamente diferentes, a um nível de significância de $1 \%$.

Após a análise descritiva, foi realizado o teste de Correlação de Pearson, com o intuito de verificar as primeiras correlações entre as variáveis do estudo, além de auxiliar na verificação do problema de multicolinearidade, pressuposto da Regressão Linear Múltipla. A Tabela 4 traz os resultados do teste.

Tabela 4 - Correlação de Pearson

\begin{tabular}{|c|c|c|c|c|c|c|c|c|c|}
\hline & $\begin{array}{c}\text { WOMEN } \\
\text { BOARD }\end{array}$ & $\begin{array}{c}\text { WOMEN } \\
\text { EXEC }\end{array}$ & TAM & BSIZE & RENT & MAS & IDV & SET & SIST \\
\hline WOMEN BOARD & 1,00 & & & & & & & & \\
\hline WOMEN EXEC & $0,27^{*}$ & 1,00 & & & & & & & \\
\hline TAM & $-0,02$ & $-0,10 * *$ & 1,00 & & & & & & \\
\hline BSIZE & 0,08 & $-0,06$ & $0,38^{*}$ & 1,00 & & & & & \\
\hline RENT & $0,08 * *$ & 0,07 & 0,00 & 0,02 & 1,00 & & & & \\
\hline MAS & $0,18^{*}$ & $0,16^{*}$ & $-0,18^{*}$ & 0,02 & $-0,14^{*}$ & 1,00 & & & \\
\hline IDV & $0,19 *$ & $-0,07$ & $0,20^{*}$ & $0,27^{*}$ & $0,14^{*}$ & $-0,50^{*}$ & 1,00 & & \\
\hline SET & $-0,09 * *$ & $-0,02$ & $-0,11^{*}$ & $-0,05$ & $-0,11 *$ & $-0,09 * *$ & $-0,04$ & 1,00 & \\
\hline SIST & $0,28^{*}$ & $-0,06$ & $0,33^{*}$ & $0,33^{*}$ & $0,11^{*}$ & $-0,13^{*}$ & $0,84 *$ & $-0,13^{*}$ & 1,00 \\
\hline
\end{tabular}

Nota: $\left({ }^{* *}\right)$ Significante ao nível de $1 \% ;\left({ }^{* *}\right)$ Significante ao nível de $5 \%$.

Fonte: Elaborada pelos autores.

Verifica-se, por meio da Tabela 4, que não há correlação superior a $50 \%$ entre as variáveis, indicando que não há alto grau de correlação, não existindo problemas elevados de multicolinearidade que possam afetar os resultados do modelo de regressão com as variáveis utilizadas.

Os resultados evidenciam correlação positiva entre as variáveis independentes rentabilidade (RENT), masculinidade (MAS) e individualismo (IDV) e participação feminina no conselho (WOMEN_BOARD). Assim, as únicas variáveis independentes que não demonstraram correlação com a diversidade de gênero no conselho foram o tamanho do conselho de administração (BSIZE) e o tamanho da empresa (TAM). A variável tamanho da empresa (TAM), entretanto, demonstrou correlação negativa com a participação feminina na alta administração (WOMEN_EXEC), enquanto a dimensão referente à masculinidade (MAS) evidenciou relação positiva com a diversidade de gênero na alta administração (WOMEN_EXEC).

Após a análise descritiva dos dados, a aplicação do teste de análise de variância e da correlação de Pearson, realizou-se o teste de Regressão Linear Múltipla, de forma a atender ao objetivo geral do estudo.

Na Tabela 5, dispõem-se os resultados da regressão, sendo testados dois modelos distintos. No Modelo 1, são avaliados os determinantes da participação feminina nas empresas, sob a ótica da participação feminina no conselho de administração. Já no Modelo 2, a variável dependente consistiu na participação feminina na alta administração das empresas. 
Francisca Everlene Nogueira Pessoa, Ana Jeniffer Rebouças Maia, Magali Carvalho Façanha, Daniel Barboza Guimarães e Silvia Maria Dias Pedro Rebouças

Tabela 5 - Regressão Linear Múltipla

\begin{tabular}{|c|c|c|c|c|c|c|c|c|}
\hline \multirow[t]{2}{*}{ Variável } & \multicolumn{4}{|c|}{ Modelo 1} & \multicolumn{4}{|c|}{ Modelo 2} \\
\hline & Beta & $T$ & Sig. & VIF & Beta & T & Sig. & VIF \\
\hline (Constante) & - & $-2,996$ & $0,003(* *)$ & - & - & $-1,696$ & 0,090 & - \\
\hline IDV & 0,358 & 3,171 & $0,002(* *)$ & 9,060 & 0,255 & 2,129 & $0,034(* *)$ & 9,060 \\
\hline MAS & 0,373 & 5,894 & $0,000\left(^{*}\right)$ & 2,847 & 0,267 & 3,979 & $0,000(*)$ & 2,847 \\
\hline TAM & $-0,026$ & $-0,555$ & 0,579 & 1,580 & $-0,012$ & $-0,249$ & 0,803 & 1,580 \\
\hline RENT & 0,077 & 2,005 & $0,045(* *)$ & 1,047 & 0,092 & 2,261 & $0,024(* *)$ & 1,047 \\
\hline BSIZE & $-0,027$ & $-0,620$ & 0,535 & 1,304 & $-0,055$ & $-1,203$ & 0,230 & 1,304 \\
\hline SET & $-0,033$ & $-0,851$ & 0,395 & 1,053 & $-0,009$ & $-0,216$ & 0,829 & 1,053 \\
\hline SIST & 0,029 & 0,290 & 0,772 & 7,228 & $-0,225$ & $-2,100$ & $0,036(* *)$ & 7,228 \\
\hline $\mathbf{R}$ & 0,391 & Durbir & -Watson & 1,771 & $\mathbf{R}$ & 0,215 & Durbin-Watson & 2,017 \\
\hline $\mathbf{R}^{2}$ & 0,153 & & $\mathbf{F}$ & 15,506 & $\mathbf{R}^{2}$ & 0,046 & $\mathbf{F}$ & 4,169 \\
\hline $\mathbf{R}^{2}$ ajustado & 0,143 & & ig. & 0,000 & $R^{2}$ ajustado & 0,035 & Sig. & 0,000 \\
\hline
\end{tabular}

Fonte: Elaborada pelos autores.

Na Tabela 5, o Modelo 1 se mostrou significativo (ao nível de 1\%), com poder explicativo de $14,3 \%$. Sobre esse modelo, foram descartados os problemas de autocorrelação dos resíduos (Durbin-Watson de 1,771). Pelo teste de Breusch-Pagan, o modelo mostrou-se homocedástico (Sig. = 0,126). As estatísticas de VIF (Variance Inflation Factor) demonstram não haver problemas significativos de multicolinearidade. A normalidade dos resíduos foi assumida pelo teorema do limite central, haja vista o considerável número de observações (STEVENSON, 2001).

Nesse sentido, constata-se que apenas as variáveis individualismo (IDV), masculinidade (MAS) e rentabilidade (RENT) se mostram significantes para explicar a participação feminina no conselho de administração das empresas, com destaque para a dimensão da cultura nacional referente à masculinidade, que apresentou influência positiva sobre a variável dependente, contrapondo-se aos resultados de alguns estudos anteriores (CARRASCO et al., 2005; HUMPHRIES; WHELAN, 2017).

O Modelo 2, por sua vez, mostrou-se significativo (ao nível de 1\%) com poder explicativo de $3,5 \%$. Foram descartados os problemas de autocorrelação dos resíduos, tendo em vista o Durbin-Watson de 2,017. O modelo mostrou-se, ainda, homocedástico (Sig. = 0,521). As estatísticas de VIF (Variance Inflation Factor) também demonstram não haver problemas significativos de multicolinearidade e, assim como no Modelo 1, a normalidade foi assumida pelo teorema do limite central, pelo considerável número de observações (STEVENSON, 2001).

Nessa perspectiva, observa-se que as variáveis individualismo (IDV) e masculinidade (MAS) e rentabilidade (RENT), de modo similar ao Modelo 1, influenciam a participação feminina na alta administração das empresas.

Destarte, rejeitam-se as hipóteses $\mathrm{H} 1$ e $\mathrm{H} 2$, de que o tamanho do conselho e o tamanho da empresa, respectivamente, relacionam-se positivamente com a participação feminina no conselho de administração e na alta administração, tendo em vista que não foram evidenciadas relações significativas entre os constructos.

Quanto à hipótese $\mathrm{H} 3$, de que a rentabilidade se relaciona positivamente com a participação feminina no conselho de administração e na alta administração, esta não pôde ser rejeitada, sinalizando que quanto maior a rentabilidade da empresa, maior a diversidade de gênero tanto no conselho de administração quanto na alta administração da empresa. Esse 
resultado corrobora os achados de Erhardt, Werbel e Shrader (2003), no contexto estadunidense, Mahadeo, Soobaroyen e Hanuman (2012), no contexto de uma economia emergente (Maurícia) e Lückerath-Rovers (2010), na Holanda.

A hipótese $\mathrm{H} 4$, que propôs relação positiva entre o individualismo e a participação feminina no conselho de administração e na alta administração, também não foi rejeitada, evidenciando que quanto mais a cultura do país no qual a empresa está inserida apresenta maior individualismo, definido por Carrasco et al. (2015) como um compromisso com os direitos humanos individuais, maior a diversidade de gênero nas empresas.

Tal resultado converge com os achados de Humphries e Whelan (2017), que pesquisaram a relação entre a cultura nacional e as recomendações de melhores práticas nos códigos de governança corporativa de 55 países. Os autores encontraram que é mais provável que os códigos de governança corporativa façam uma recomendação relacionada à composição de gênero do conselho em países com altos índices de individualismo.

Por fim, rejeita-se a hipótese $\mathrm{H} 5$, de que uma cultura nacional de elevada masculinidade se relaciona negativamente com a participação feminina no conselho de administração e na alta administração. Diferente da relação negativa prevista, os resultados evidenciaram uma relação positiva entre a masculinidade e a diversidade de gênero. Isto significa que o fato de os países atribuírem valor significativo à competitividade, ao sucesso e à determinação gerencial, o que caracteriza a dimensão chamada explicitada no website de Hofstede (2017) como masculinidade, influencia positivamente a diversidade de gênero das empresas.

Este achado diverge dos resultados de Carrasco et al. (2005), que investigaram a representação feminina no conselho em 32 países, relacionando às dimensões culturais de Hofstede (1980), tendo como resultado que a masculinidade estava relacionada negativamente à participação feminina. De modo similar, diverge do resultado encontrado por Humphries e Whelan (2017), que evidenciaram que países com altos índices de masculinidade são menos propensos a ter códigos de governança corporativa que exigem diversidade de gênero.

Entretanto, esse resultado pode ser justificado pelo fato de que as diferentes características que se atribuem ao sexo feminino provêm, em grande parte, de estereótipos (BAUER, 2015; EDDLESTON; POWELL, 2008). Ademais, na perspectiva da teoria do teto de vidro, as mulheres que rompem as barreiras para alcançar a alta gerência podem adaptar o seu comportamento, em um ambiente predominantemente masculino, para que as diferenças de gênero desapareçam (ADAMS, FUNK, 2012).

Nesse cenário, a relação positiva encontrada entre a masculinidade e a participação feminina no conselho e alta administração corrobora a teoria do teto de vidro, pela qual as mulheres se adequam aos valores masculinos para alcançar cargos superiores, e incita a reflexão de até que ponto essa maior participação reflete a diversidade e equidade na divisão do trabalho.

Assim, das conclusões que podem ser obtidas, observa-se que a rentabilidade e a cultura nacional, representada pelas dimensões de individualismo e masculinidade, demonstraram ser fatores determinantes da diversidade de gênero, considerando tanto a participação feminina no conselho de administração, quanto na alta administração das empresas. 
Francisca Everlene Nogueira Pessoa, Ana Jeniffer Rebouças Maia, Magali Carvalho Façanha, Daniel Barboza Guimarães e Silvia Maria Dias Pedro Rebouças

\section{CONSIDERAÇÕES FINAIS}

Considerando a representatividade feminina nas empresas, haja vista o crescente envolvimento das mulheres nos negócios e a escassez de estudos abordando a temática no BRICS, esta pesquisa teve como objetivo principal identificar os determinantes da diversidade de gênero em empresas do BRICS. Para tanto, o estudo foi realizado com uma amostra de 611 empresas por meio de análises descritivas, análise de variância, testes de correlação e regressão linear múltipla, considerando o exercício de 2016.

Com a análise descritiva, verificou-se que as médias da participação feminina nas empresas refletem uma baixa diversidade de gênero, existindo empresas sem representação feminina no Brasil, China, Índia e Rússia. O país que apresentou maior diversidade de gênero no conselho de administração, bem como na alta administração das empresas, foi a África do Sul, enquanto os países com menor diversidade de gênero nas empresas foram Rússia e Índia.

A análise de variância, por sua vez, demonstrou a existência de diferenças significativas na participação feminina das empresas entre os países, tanto em relação à proporção de mulheres no conselho de administração quanto na alta administração das empresas, reforçando a relevância do estudo abrangendo empresas de diferentes países.

Dentre os principais resultados da Correlação de Pearson, ressalta-se que o individualismo, a masculinidade e a rentabilidade apresentaram correlação positiva com a participação feminina no conselho, enquanto o tamanho da empresa apresentou correlação negativa com a participação feminina na alta administração e a masculinidade evidenciou correlação positiva com a diversidade de gênero na alta administração.

Por meio da Regressão Linear Múltipla, que buscou atender ao objetivo geral de pesquisa, constatou-se que a rentabilidade e a cultura nacional, representada pelas dimensões de individualismo e masculinidade, são determinantes da diversidade de gênero no conselho de administração e na alta administração das empresas.

Destarte, o trabalho põe em evidência a diversidade de gênero nas empresas e contribui para a discussão acerca dos fatores determinantes da participação feminina no conselho de administração e na alta administração das empresas, fornecendo evidências empíricas da análise para países de economias emergentes.

Nesse sentido, as contribuições do presente estudo consistem na observação da participação feminina nas empresas por mais de uma ótica: não apenas no conselho de administração, órgão que representa a governança corporativa das empresas, mas ainda na alta administração, considerando a gestão das empresas.

Os resultados apresentados implicam na constatação da, embora crescente, baixa representatividade feminina na governança corporativa e na gestão das empresas pertencentes a países de economias emergentes, preenchendo uma lacuna na literatura acerca dos determinantes da diversidade de gênero nesse contexto.

Ademais, os resultados do estudo incitam a reflexão acerca da diversidade de gênero nas organizações, tendo em vista que a presença feminina na governança e gestão das empresas pode ocorrer em virtude da adequação das mulheres aos comportamentos masculinos, não representando, dessa forma, a diversidade e a eliminação da divisão sexual do trabalho.

Os achados deste estudo são limitados às empresas da amostra dentro do período analisado. Assim, sugere-se para estudos futuros uma análise com maior quantidade de países, a fim de possibilitar uma análise comparativa, além de uma análise longitudinal, propiciando a observação do efeito temporal dos determinantes da diversidade de gênero nas 
empresas, bem como a ampliação das formas de mensuração da diversidade de gênero, com vistas a refletir a igualdade de trabalho entre homens e mulheres no meio organizacional.

\section{REFERÊNCIAS}

ADAMS, R.; FERREIRA, D. A theory of friendly boards. The Journal of Finance, v. 62, n. 1, p. 217-250, 2007. DOI: https://doi.org/10.1111/j.1540-6261.2007.01206.x

ADAMS, R. B.; FERREIRA, D. Women in the boardroom and their impact on governance and performance. Journal of Financial Economics, v. 94, n. 2, p. 291-309, 2009. DOI: https://doi.org/10.1016/j.jfineco.2008.10.007

ADAMS, R. B.; FUNK, P. Beyond the Glass Ceiling: Does Gender Matter? Management Science, v. 58, n. 2, p. 219-235, 2012. DOI: http://dx.doi.org/10.1287/mnsc.1110.1452

ALI, M. Determinants and consequences of board size: conditional indirect effects. Corporate Governance, v. 18, n. 1, p. 165-184, 2018. DOI: https://doi.org/10.1108/CG-012016-0011

ARFKEN, D. E.; BELLAR, S. L.; HELMS, M. M. The ultimate glass ceiling revisited: The presence of women on corporate boards. Journal of Business ethics, v. 50, n. 2, p. 177-186, 2004. DOI: https://doi.org/10.1023/B:BUSI.0000022125.95758.98

ARTHUR, J. B.; HERDMAN, A. O.; YANG, J. How Top Management HR Beliefs and Values Affect High-Performance Work System Adoption and Implementation Effectiveness. Human Resource Management, v. 55, n. 3, p. 413-435, 2016. DOI:

https://doi.org/10.1002/hrm.21672

BAUER, N. M. Who stereotypes female candidates? Identifying individual differences in feminine stereotype reliance. Journal Politics, Groups, and Identities, v. 3, n. 1, p. 94-110, 2015. DOI: https://doi.org/10.1080/21565503.2014.992794

BERNILE, G.; BHAGWAT, V.; YONKER, S. Board diversity, firm risk, and corporate policies. Journal of Financial Economics, v. 127, n. 3, p. 588-612, 2018. DOI: https://doi.org/10.1016/j.jfineco.2017.12.009

BOULOUTA, I. Hidden Connections: The Link Between Board Gender Diversity and Corporate Social Performance. Journal of Business Ethics, v. 113, p. 185-197, 2013. DOI: https://doi.org/10.1007/s10551-012-1293-7

BRAMMER, S.; MILLINGTON, A.; PAVELIN, S. Gender and ethnic diversity among UK corporate boards. Corporate Governance: An International Review, v. 15, n. 2, p. 393-403, 2007. DOI: https://doi.org/10.1111/j.1467-8683.2007.00569.x

BUCKALEW, E.; KONSTANTINOPOULOS, A.; RUSSELL, J.; EL-SHERBINI, S. The Future of Female CEOs and Their Glass Ceiling. Journal of Business Studies Quarterly, v. 3, n. 4, p.145-153, 2012. 
Francisca Everlene Nogueira Pessoa, Ana Jeniffer Rebouças Maia, Magali Carvalho Façanha, Daniel Barboza Guimarães e Silvia Maria Dias Pedro Rebouças

BURKE, R. J. Women Directors: Selection, Acceptance and Benefits of Board Membership. Corporate Governance: An International Review, v. 5, p. 118-125, 1997. DOI: https://doi.org/10.1111/1467-8683.00052

BURKE, R.; NELSON, D. Advancing Women in Management: Progress and Prospects. In: BURKE, R.; NELSON, D. (eds). Advancing Women's Careers. Oxford: Blackwell, p. 3-14, 2002.

CARRASCO, A.; FRANCOEUR, C.; LABELLE, R.; LAFFARGA, J.; RUIZ-

BARBADILLO, E. Appointing Women to Boards: Is There a Cultural Bias? Journal of Business Ethics, v. 129, n. 2, p. 429-444, 2015. DOI: https://doi.org/10.1007/s10551-014-2166-z

CARTER, D. A.; SIMKINS, B. J.; SIMPSON, W. G. Corporate governance, board diversity, and firm value. The Financial Review, v. 38, p. 33-53, 2003. DOI: https://doi.org/10.1111/15406288.00034

CATALYST. Catalyst census of women board directors of the Fortune 500. 2008.

COMISSÃO EUROPEIA. Women on boards - Factsheet 1 The economic arguments. 2012.

COOK, A.; GLASS, C. Above the glass ceiling: When are women and racial/ethnic minorities promoted to CEO? Strategic Management Journal, v. 35, n. 7, p. 1080-1089, 2013. DOI: https://doi.org/10.1002/smj.2161

DAVIDSON, M. J.; BURKE, R. J. Women in management worldwide: facts, figures and analysis-an overview. In: DAVIDSON, M. J.; BURKE, R. J. (Eds). Women in Management Worldwide: Facts, Figures and Analysis. Ashgate Publishing Limited, London, p. 1-15, 2004.

DREHER, G. F. Breaking the Glass Ceiling: The Effects of Sex Ratios and Work-Life Programs on Female Leadership at the Top. Human Relations, v. 56, n. 5, p. 541-562, 2003. DOI: https://doi.org/10.1177/0018726703056005002

DU, X. Does confucianism reduce board gender diversity? Firm-level evidence from China. Journal of Business Ethics, v. 136, n. 2, p. 399-436, 2016. DOI: https://doi.org/10.1007/s10551-014-2508-x

EAGLY, A. The his and hers of prosocial behavior: An examination of the social psychology of gender. American Psychologist, v. 64, p. 644-658, 2009. DOI: https://doi.org/10.1037/0003066X.64.8.644

EAGLY, A. H.; WOOD, W. Social Role Theory of Sex Differences. In: Naples N, Hoogland RC, Wickramasinghe M, Wong WCA (Eds.), The Wiley Blackwell Encyclopedia of Gender and Sexuality Studies. 2016.

EDDLESTON, K. A.; POWELL, G. N. The role of gender identity in explaining sex differences in business owners' career satisfier preferences. Journal of Business Venturing, v. 23, n. 2, p. 244-256, 2008. DOI: https://doi.org/10.1016/j.jbusvent.2006.11.002 
ELYASIANI, E.; ZHANG, L. Bank holding company performance, risk, and "busy" board of directors. Journal of Banking \& Finance, v. 60, n. 1, p. 239-251, 2015. DOI: https://doi.org/10.1016/j.jbankfin.2015.08.022

ERHARDT, N. L.; WERBEL, J. D.; SHRADER, C. B. Board of director diversity and firm financial performance. Corporate Governance, v. 11, n. 2, p. 102-111, 2003. DOI: https://doi.org/10.1111/1467-8683.00011

EZZEDEEN, S. R.; BUDWORTH, M.-H.; BAKER, S. D. The Glass Ceiling and Executive Careers: Still an Issue for Pre-Career Women. Journal of Career Development, v. 42, n. 5, p. 355-369, 2015. DOI: https://doi.org/10.1177/0894845314566943

FARRELL, K. A.; HERSCH, P. L. Additions to corporate boards: the effect of gender. Journal of Corporate Finance, v. 11, n. 1-2, p. 85-106, 2005. DOI: https://doi.org/10.1016/j.jcorpfin.2003.12.001

FORTIN, N. M. Gender Role Attitudes and Women's Labor Market Participation: Opting-Out, AIDS, and the Persistent Appeal of Housewifery. Economics and Statistics, n. 117/118, p. 379-401, 2015. DOI: https://doi.org/ 10.15609/annaeconstat2009.117-118.379

GÁMEZ, A. E.; ANGELES, M. Women's Participation in the Labor Market. In: LEAL FILHO, W. et al. (eds.), Gender Equality. Encyclopedia of the UN Sustainable Development Goals, 2019. DOI: https://doi.org/10.1007/978-3-319-70060-1_3-1

GÓMEZ, J. M.; LAFUENTE, E.; VAILLANT, Y. Gender diversity in the board, women's leadership and business performance. Gender in Management, v. 33, n. 2, p. 104-122, 2018. DOI: https://doi.org/10.1108/GM-05-2017-0058

GONÇALVES, E. B. P. ESPEJO, M. M. S. B.; ALTOÉ, S. M. L.; VOESE, S. B. Gestão da diversidade: um estudo de gênero e raça em grandes empresas brasileiras. Enfoque: Reflexão Contábil, v. 35 , n. 1, p. 95-112, 2016. DOI: 10.4025/enfoque.v35i1.30050

GREEN, C. P.; HOMROY, S. Female directors, board committees and firm performance. European Economic Review, v. 102, p. 19-38, 2018. DOI: https://doi.org/10.1016/j.euroecorev.2017.12.003

GULL, A. A.; NEKHILI, M.; NAGATI, H.; CHTIOUI, T. Beyond gender diversity: How specific attributes of female directors affect earnings management. The British Accounting Review, v. 50, n. 3, p. 255-274, 2018. DOI: https://doi.org/10.1016/j.bar.2017.09.001

HOFHUIS, J.; ZEE, K. I. V. D.; OTTEN, S. Measuring employee perception on the effects of cultural diversity at work: development of the Benefits and Threats of Diversity Scale. Quality \& Quantity, v. 49, p. 177-201, 2015. DOI: https://doi.org/10.1007/s11135-013-99817 
Francisca Everlene Nogueira Pessoa, Ana Jeniffer Rebouças Maia, Magali Carvalho Façanha, Daniel Barboza Guimarães e Silvia Maria Dias Pedro Rebouças

HUMPHRIES, S. A.; WHELAN, C. National culture and corporate governance codes. Corporate Governance: The International Journal of Business in Society, v. 17, n. 1, p. 152-163, 2017. DOI: https://doi.org/10.1108/CG-06-2016-0127

HYLAND, M.; MARCELLINO, P. Examining gender on corporate boards: a regional study. Corporate Governance, v. 2, n. 4, p. $24-31$, 2002. DOI: https://doi.org/10.1108/14720700210447669

JOGULU, U. D.; WOOD, G. J. A cross-cultural study into peer evaluations of women's leadership effectiveness. Leadership \& organization development journal, v. 29, n. 7, p. 600-616, 2008. DOI: https://doi.org/10.1108/01437730810906344

KAKABADSE, C. F. N. K.; NICOLOPOULOU, J. H. Y. K.; KAKABADSE, M. F. O. A. Gender Diversity and Board Performance: Women's Experiences and Perspectives. Human Resource Management, v. 55, n. 2, p. 265-281, 2015. DOI: https://doi.org/10.1002/hrm.21694

KANG, H.; CHENG, M.; GRAY, S. J. Corporate governance and board composition: Diversity and independence of Australian Boards. Corporate Governance: An International Review, v. 15, n. 2, p. 194-207, 2007. DOI: https://doi.org/10.1111/j.1467-8683.2007.00554.x

LA PORTA, R.; LOPEZ-DE-SILANES, F.; SHLEIFER, A. Corporate ownership around the world. Journal of Finance, v. 54, p. 471-517, 1999. DOI: https://doi.org/10.1111/0022-1082.00115

LAZZARETTI, K.; GODOI, C. K. A participação feminina nos conselhos de administração das empresas brasileiras: uma análise das características de formação acadêmica e experiência profissional à luz da teoria do capital humano. Revista Gestão \& Conexões, v. 1, n. 1, 2012.

LEHN, K. M.; PATRO, S.; ZHAO, M. Determinants of the Size and Composition of US Corporate Boards: 1935-2000. Financial Management, v. 38, n. 4, p. 747-780, 2009. DOI: https://doi.org/10.1111/j.1755-053X.2009.01055.x

LIAO, L.; LUO, L.; TANG, Q. Gender diversity, board independence, environmental committee and greenhouse gas disclosure. The British Accounting Review, v. 47, n. 4, p. 409-424, 2015. DOI: https://doi.org/10.1016/j.bar.2014.01.002

LI, H.; CHEN, P. Board gender diversity and firm performance: The moderating role of firm size. Business Ethics: A European Review, v. 27, n. 4, p. 294-308, 2018. DOI: https://doi.org/10.1111/beer.12188

LÜCKERATH-ROVERS, M. A Comparison of Gender Diversity in the Corporate Governance Codes of France, Germany, Spain, the Netherlands and the United Kingdom. 2010. Working Paper.

LUOMA, P.; GOODSTEIN, J. Stakeholders and Corporate Boards: Institutional Influences on Board Composition and Structure. Academy of Management Journal, v. 42, n. 5, p. 553, 1999. DOI: https://doi.org/10.5465/256976 
MAHADEO, J. D.; SOOBAROYEN, T.; HANUMAN, V. O. Board Composition and Financial Performance: Uncovering the Effects of Diversity in an Emerging Economy. Journal of Business Ethics, v. 105, n. 3, p. 375-388, 2012. DOI: https://doi.org/10.1007/s10551-0110973-z

MARTINS, G. A.; THEÓPHILO, C. R. Metodologia da investigação científica para ciências sociais aplicadas. 2. ed. São Paulo: Atlas, 2009.

MATTIS, M. C. Women Corporate Directors in the United States. In: BURKE, R.; MATTIS, M. (eds). Women on Corporate Boards of Directors. Netherlands: Kluwer Academic, 2000.

MEISSNER, F.; VERTOVEC, S. Comparing super-diversity. Ethnic and Racial Studies, v. 38, n. 4, p. 541-55, 2015. DOI: https://doi.org/10.1080/01419870.2015.980295

NIELSEN, S.; HUSE, M. The Contribution of Women on Boards of Directors: Going beyond the Surface. Corporate Governance: An International Review, v. 18, n. 2, p. 136-148, 2010. DOI: https://doi.org/10.1111/j.1467-8683.2010.00784.x

NOGUEIRA, E. C. O. R.; KUBO, E. K. M. Sentidos do exercício da liderança por mulheres executivas brasileiras. Revista de Gestão e Secretariado, v. 4, n. 2, p. 114-133, 2013. DOI: https://doi.org/10.7769/gesec.v4i2.175

OAKLEY, J. Gender-based barriers to senior management positions: Understanding the scarcity of female CEOs. Journal of Business Ethics, v. 27, n. 4, p. 321-334, 2000. DOI: https://doi.org/10.1023/A:1006226129868

PATRÍCIO, S. S. S. Os determinantes das mulheres no conselho de administração: o caso das sociedades anónimas portuguesas. 2014. Dissertação (Mestrado em Contabilidade, Fiscalidade e Finanças Empresariais) - Lisboa School of Economics and Management, Lisboa, 2014.

PELLED, L. Demographic Diversity, Conflict, and Work Group Outcomes: An Intervening Process Theory. Organization Science, v. 7, p. 615-631, 1996. DOI: https://doi.org/10.1287/orsc.7.6.615

PENG, M. W. Outside directors and firm performance during institutional transitions. Strategic Management Journal, v. 25, p. 453, 2004. DOI: https://doi.org/10.1002/smj.390

PERETZ, H.; LEVI, A.; FRIED, Y. Organizational diversity programs across cultures: effects on absenteeism, turnover, performance and innovation. The International Journal of Human Resource Management, v. 26, n. 6, p. 875-903, 2015. DOI: https://doi.org/10.1080/09585192.2014.991344

PERRYMAN, A. A.; FERNANDO, G. D.; TRIPATHY, A. Do gender differences persist? An examination of gender diversity on firm performance, risk, and executive compensation. Journal of Business Research, v. 69, n. 2, p. 579-586, 2016. DOI: https://doi.org/10.1016/j.jbusres.2015.05.013 
Francisca Everlene Nogueira Pessoa, Ana Jeniffer Rebouças Maia, Magali Carvalho Façanha, Daniel Barboza Guimarães e Silvia Maria Dias Pedro Rebouças

PFEFFER, J.; SALANCIK, G. R. The external control of organizations: A resource dependence perspec-tive. New York: Harper \& Row, 1978.

POWELL, W. W. Expanding the scope of institutional analysis. In: POWELL, W. W.; DIMAGGIO, P. J. (Eds.). The new institutionalism in organizational analysis. Chicago: University of Chicago Press, 1991.

SALANCICK, G. R. Interorganizational Dependence and Responsiveness to Affirmative Action: The Case of Women and Defense Contractors. Academy of Management Journal, v. 22, n. 2, p. 375-394, 1979. DOI: https://doi.org/10.5465/255596

SAMPIERI, R. H.; COLLADO, C. F.; LÚCIO, M. P. B. Metodologia de pesquisa. 5. ed. São Paulo: McGraw Hill, 2013.

SANTOS, E. S.; VIANA JR, D. B. C. Explanatory Factors of Corporate Governance Disclosure in OECD and BRICS Countries. In: Encontro da Associação Nacional de Pós-Graduação e Pesquisa em Administração - EnANPAD, XLI, São Paulo. Anais [...]. São Paulo: ANPAD, 2017. Disponível em:

http://www.anpad.org.br/eventos.php?cod_evento=1\&cod_edicao_subsecao=1453\&cod_e vento_edicao $=89 \&$ cod_edicao_trabalho $=23129$

SEALY, R.; DOLDOR, E.; VINNICOMBE, S. Increasing diversity on public and private sector boards. Part 1: How diverse are boards and why? Cranfield School of Management Report commissioned by the UK Government Equalities Office, 2009.

SILA, V.; GONZALEZ, A.; HAGENDORF, J. Women on board: Does boardroom gender diversity affect firm risk? Journal of Corporate Finance, v. 36, p. 26-53, 2016. DOI:

https://doi.org/10.1016/j.jcorpfin.2015.10.003

SILVA, A. L. C.; MARGEM, H. Mulheres em Cargos de Alta Administração Afetam o Valor e Desempenho das Empresas Brasileiras? Revista Brasileira de Finanças, v. 13, n. 1, p. 103133, 2015.

SILVA JÚNIOR, C. P.; MARTINS, O. S. Mulheres no Conselho Afetam o Desempenho Financeiro? Uma Análise da Representação Feminina nas Empresas Listadas na BM\&FBovespa. Sociedade, Contabilidade e Gestão, v. 12, n. 1, p. 62-76, 2017.

SILVEIRA, A.; DONAGGIO, A.; SICA, L.; RAMOS, L. Women's participation in senior management positions: gender social relations, law and corporate governance. 2014. Working Paper.

SOLAKOGLU, M. N.; DEMIR, N. The role of firm characteristics on the relationship between gender diversity and firm performance. Management Decision, v. 54, n. 6, p. 1.407-1419, 2016. DOI: https://doi.org/10.1108/MD-02-2015-0075

STEVENSON, W. J. Estatística aplicada à administração. São Paulo: Harba, 2001. 
TERJESEN, S.; SEALY, R.; SINGH, V. Women directors on corporate boards: A review and research agenda. Corporate governance: an international review, v. 17, n. 3, p. 320-337, 2009. DOI: https://doi.org/10.1111/j.1467-8683.2009.00742.x

VIANA D. B. C., JR., VASCONCELOS, A. C., DE LUCA, M. M. M.; PONTE, V. M. R. Determinantes do disclosure de governança corporativa: Evidências em empresas de quatro países latinoamericanos. In: Encontro da Associação Nacional de Pós-Graduação e Pesquisa em Administração - EnANPAD, XLI, São Paulo. Anais [...]. São Paulo: ANPAD, 2017.

WADDOCK, S. A.; GRAVES, S. B. The corporate social performance-financial performance link. Strategic Management Journal, v. 18, p. 303-319, 1997. DOI:

https://doi.org/10.1002/(SICI)1097-0266(199704)18:4<303::AID-SMJ869>3.0.CO;2-G 